\title{
Solving ill-posed magnetic inverse problem using a Parameterized Trust-Region Sub-problem
}

\author{
Maha ABDELAZEEM Mohamed ${ }^{1}$ \\ ${ }^{1}$ National Research Institute of Astronomy and Geophysics (NRIAG) \\ Helwan, Cairo, Egypt; e-mail: maazeem03@yahoo.com
}

\begin{abstract}
The aim of this paper is to find a plausible and stable solution for the inverse geophysical magnetic problem. Most of the inverse problems in geophysics are considered as ill-posed ones. This is not necessarily due to complex geological situations, but it may arise because of ill-conditioned kernel matrix. To deal with such ill-conditioned matrix, one may truncate the most ill part as in truncated singular value decomposition method (TSVD). In such a method, the question will be where to truncate? In this paper, for comparison, we first try the adaptive pruning algorithm for the discrete L-curve criterion to estimate the regularization parameter for TSVD method. Linear constraints have been added to the ill-conditioned matrix. The same problem is then solved using a global optimizing and regularizing technique based on Parameterized Trust Region Sub-problem (PTRS). The criteria of such technique are to choose a trusted region of the solutions and then to find the satisfying minimum to the objective function. The ambiguity is controlled mainly by proper choosing the trust region. To overcome the natural decay in kernel with depth, a specific depth weighting function is used. A Matlab-based inversion code is implemented and tested on two synthetic total magnetic fields contaminated with different levels of noise to simulate natural fields. The results of PTRS are compared with those of TSVD with adaptive pruning L-curve. Such a comparison proves the high stability of the PTRS method in dealing with potential field problems. The capability of such technique has been further tested by applying it to real data from Saudi Arabia and Italy.
\end{abstract}

Key words: inversion, ill-posed problem, ill-conditioned, Tikhonov regularization, Trust Region Sub-problem, TRS, Parameterized Trust Region Sub-problem, PTRS, depth weighting

\section{Introduction}

Inverse potential field problems, which we aim to solve here, represent typical ill-posed problems. This is due to the invariable contamination of data with 
noise and such data is acquired in a limited number of observation points. These problems are said to be ill-posed or ill-conditioned, which means that the solutions are non-unique and unstable. Most literature reformulated such problems into a system of equations having better condition by adding different kinds of constraints to control the results as much as possible. For example, Last and Kubik (1983) imposed a condition that the volume of the causative body to be minimum and have a definite upper boundary for the unknown physical property. Guillen and Menichetti (1984) constrained the source to have minimal moment of inertia with respect to the center of gravity or to an axis of given dip passing through it. Abdelazeem et al. (1998) used a quadratic programming with both equality and inequality constraints to invert the gravity anomalies.

We investigated the synthetic models of Last and Kubik (1983) carefully and it is found that all data kernel matrices have low condition numbers. When the same subsurface models were taken in different numbers of rows and/or columns, their technique failed to invert; this is because such matrices have higher condition numbers than the original ones. Such cases can now be classified as ill-posed/ill-conditioned problems. Thence, it is obvious that in ill-posed problems, a proper solution will not be achieved even when using constraints if traditional techniques are utilized.

Therefore, to solve these problems we need special strategies known as regularization techniques. To regularize such a problem, at first, the illposedness of the problem has to be tested by calculating the condition number of the kernel matrix. The higher the condition number, the higher the ill-posedness of the problem. Secondly, any conventional regularization tool can be used in order to have acceptable trusted solutions (Tikhonov and Arsenin, 1977). Recently, such a problem is tackled using different forms (Zhdanov, 1993). Tezkan et al. (2000) used regularization with conjugate gradient to invert radiomagnetotelluric field to delineate industrial and domestic waste sites in Germany. Portniaguine and Zhdanov (2002) developed a regularization technique, based on the traditional Tikhonov regularization theory, by using a weighted model parameters based on sensitivity analysis and fine focused inversion and applied it to 3-D magnetic problems. However, they didn't mention what will be the case if data were contaminated with high levels of noise. Fedi et al. (2005) presented a new tool, the Depth Resolution Plot (DRP) for regularizing inverse potential 
field problems. Silva et al. (2007) used the entropic regularization to estimate the density contrast of elongated prismatic cell with known tops and bottoms. Abdelazeem et al. (2007) used TSVD with adaptive pruning L-curve technique to invert the ill-posed gravity problem. Blaschek et al. (2008) used a new regularization scheme based on the Minimum Gradient Support (MGS) and active constraint balancing to invert the induced polarization data. Ardalan et al. (2011) used an alternative method for density variation modeling of the crust from constrained inversion of the terrestrial gravity data. Pašteka et al. (2012) used Tikhonov regularization for stable downward continuation of geophysical potential fields.

To summarize, the magnetic inverse problem is, intrinsically, non-unique and its numerical solution is unstable. This means that any small perturbation in the data (noise) causes large variation in the solution. Such problem is a highly ill-posed one. We applied (Grodzevich, 2004) regularization technique for formulating generalized solutions that are stable even with perturbed data. The method is an extension to the traditional trustregion approach combined with L-curve. The regularization parameter is deduced during inversion. The radius of the trust region is changed during iteration; to improve the solution. The method has been applied to invert two synthetic examples with different levels of noise in order to test its stability in highly perturbed data condition. The method is compared to TSVD with adaptive pruning L-curve (Hansen et al., 2007), which was applied successfully to invert the gravity inverse problem (Abdelazeem, 2007). Finally, the method has been applied to a total field anomaly from Saudi Arabia and a vertical gradient field from Italy in order to test its validity.

\section{Ill-posed problem}

To clarify the source of ill-posedness of our problem and why the regular inversion tools are not the optimum choice, let us begin from the initial description of the magnetic field. The magnetic field is expressed (Blakley, 1996) in an integral equation of the form

$f(P)=\int_{R} s(Q) \psi(P, Q) \mathrm{d} v$ 
where $f(P)$ is the magnetic field at $P$ (the observation point), $s(Q)$ describes the magnetization at $Q, \psi(P, Q)$ is a function that depends on the geometric placement of observation point $P$ and source point $Q . R$ is the volume/area occupied by the causative source and $Q$ is the point of integration within $R$. This equation is known as Fredholm integral of the first kind (Morse and Feshbach, 1953). Now to study this problem, the subsurface domain is divided into a set of $M$ rectangular prismatic cells. Each cell has a constant susceptibility of $s$ value, assuming that there is no remnant magnetization and the demagnetization effect is negligible. Thus, only the induced magnetization is considered. Using the discrete quadrature rule for discretizing such integration (Baker, 1977), the above integral equation is approximated as follows:

$\int_{a}^{b} \int_{c}^{d} \psi(P, Q) s(Q) \mathrm{d} P \mathrm{~d} Q \cong \sum_{i=1}^{N} \sum_{j=1}^{M} w_{i} w_{j}^{\prime} \psi\left(P_{i}, Q_{j}\right) s\left(Q_{j}\right)=f\left(p_{i}\right)$,

where $s\left(Q_{j}\right), \psi\left(P_{i}, Q_{j}\right)$ and $f\left(P_{i}\right)$ are the approximation of $s(Q), \psi(P, Q)$ and $f(P)$ respectively, $w_{i}=\frac{b-a}{N}$ and $w_{j}^{\prime}=\frac{d-c}{M}$, where $[a, b]$ is the outer integral interval, $[c, d]$ is the inner integral interval, $N$ is the number of data points and $\mathrm{M}$ is the number of prisms. When equation (2) is written in matrix notation, one obtains the system: $G_{N \times M} s_{M}=D_{N}$, where $G_{N \times M}$ is an $N \times M$ matrix, which quantifies the effect of the $j^{\text {th }}$ cell on the $i^{\text {th }}$ data point. The elements of $G, s$ and D are given by $g_{i j}=w_{i} w_{j}^{\prime} \psi_{i j}\left(P_{i}, Q_{j}\right)$, $D_{i}=f\left(P_{i}\right), s_{j}=s\left(Q_{j}\right), \mathrm{i}=1,2, ., N$ and $\mathrm{j}=1,2, . ., M$. As data is always contaminated with noise, we will consider the measured data $\bar{D}=D+\eta$, where $\eta$ is a random vector of uncorrelated noise. Then, the problem to be solved is:

$\min _{\mathrm{s}}\|G s-\bar{D}\|^{2}$

Using a reasonably accurate discretization to obtain $G$ will result in a highly ill-conditioned matrix with a singular spectrum that decays to zero gradually, a large cluster of small singular values, and high-frequency components of the singular vectors associated with small singular values (Rojas and Sorensen, 2002). If, in addition, the Discrete Picard Condition (Hansen, 1990) holds, the expansion coefficients of the exact data vector $D$ will decay to zero faster than the singular values of $G$, while the expansion coefficients 
of the noise vector $\eta$ remains constant. Therefore, the solutions corresponding to small singular values are magnified by the noise. As a consequence of the ill conditioning of the matrix $G$ and the presence of noise in data, applying standard numerical methods such as those discussed in Dennis and Schnabel (1983) to Eq. 3 will produce meaningless solutions with very large norm. Therefore, to solve these problems, we need special techniques known as regularization or smoothing methods. Such methods focus on recovering information about the desired solution from the solution of a better conditioned problem that is related to the problem with noisy data but incorporates additional information about the desired solution. The formulation of the new problem involves a special parameter (the regularization parameter), used to control the effect of the noise on the solution. The conditioning of the new problem depends on the choice of the regularization parameter. Good surveys on regularization methods can be found for example in (Zhdanov, 1993 and Hansen, 1998).

In the next two sections, we will give a brief introduction to the concepts of regularization strategies employed for solving our ill-posed problem.

\section{Method}

In order to introduce the method applied in this paper, we have to mention some essential concepts used. Firstly, the classical Tikhonov regularization form, which is the base for most regularizing tools. Secondly, the trust region sub-problem (TRS) form, which is the base also for many regularizing tools and for the main tool used here. Thirdly, the L-curve criterion which is sometimes implemented with other tools to choose the optimum regularized solution. Lastly, we will mention the Picard condition and its use in testing the ill-posedness of the problem. Then, the main tool used, Parameterized Trust Region Sub-problem, PTRS method (Grodzevich, 2004) is presented. Another method is presented to be applied here, in order to clarify the strength of the PTRS, named TSVD with adaptive pruning Lcurve (Hansen et al., 2007).

\subsection{Tikhonov and TRS forms}

The classical Tikhonov regularization approach is one of the most popular regularization approaches for solving discrete forms of ill-conditioned linear 
algebraic system given in Eq. 3. Tikhonov regularization problem takes the form

$\left(G^{T} G+\alpha^{2} I\right) s_{\alpha}=G^{T} \bar{D}$,

where $\alpha$ is the regularization parameter and $s_{\alpha}$ is the regularized solution. The success of the Tikhonov regularization technique depends on making a good choice of the regularization parameter $\alpha$, which is not easy to find. The reason is that the solution $s_{\alpha}$ is too sensitive to perturbations in $D$, i.e., a small change in $D$ may produce a large change in $s_{\alpha}$.

On the other hand, it is well known that a trust-region sub-problem (TRS) method is quite attractive optimization technique, which finds a direction and a step size in an efficient and reliable manner with the help of a quadratic model of the objective function (Gander, 1978). In general, the trust-region methods define a region around the current iteration within which they trust the model to be an adequate representation of the objective function, and then choose the step to be the approximate minimizer of the model in this trust-region. In effect, they choose the direction and length of the step simultaneously. If a step is not acceptable, they reduce the size of the region and find a new minimizer. The step direction changes whenever the size of the trust-region is altered. To approach the TRS form, we should begin with the constrained least-squares problem, which takes the form

$\min _{s}\|G s-\bar{D}\|_{2}$, subject to $\|s\|_{2} \leq \varepsilon$.

Regularization depends here on choosing the parameter $\varepsilon$. The problem can be re-formulated as $\mu(A, a, \varepsilon)$ the so called trust-region sub-problem TRS (Fortin and Wolkowics, 2004) as:

$\mu(A, a, \varepsilon) \quad \min q(s):=s^{T} A s-2 a^{T} s$, subject to $\|s\|_{2}^{2} \leq \varepsilon^{2}$,

where $A:=G^{T} G$ is a $M \times M$ symmetric $(m>2), a:=G^{T} \bar{D}$ is an m-vector. $\varepsilon$ is the trust-region's fixed radius (positive scalar), and $s$ is the m-vector of unknowns. All matrix and vector entries are real.

\subsection{L-curve criterion and Picard condition}

The L-curve criterion is based on a log-log plot of the corresponding values of the residual and solution norms $\left(\log \left\|G s_{k}-\bar{D}\right\|_{2}, \log \left\|s_{k}\right\|_{2}\right), k=1, \ldots, p$. 
The optimal regularization parameter corresponds to a point near the corner. Singular Value Decomposition (SVD) of the matrix $G$ is a tool that helps in understanding the L-curve analysis. It can be written as:

$\mathrm{G}=\mathrm{USV}^{\mathrm{T}}$,

where matrix $\mathrm{S}$ is a diagonal $n \times n$ matrix consisting of singular values $\sigma_{\mathrm{i}}$ of $\mathrm{G}, \sigma_{1} \geq \ldots \geq \sigma_{\mathrm{n}}$ and $\mathrm{U}, \mathrm{V}$ are orthogonal matrices, i.e:

$U^{T} U=I, \quad V^{T} V=I$.

So, the Tikhonov regularized solution $s_{\alpha}$ can be written by substituting the SVD of matrix G into Eq. 4 as:

$\left\|s_{\alpha}\right\|_{2}^{2}=\bar{D}^{T} U\left(S\left(S^{2}+\alpha^{2} I\right)\right)^{2} U^{T} \bar{D}=\sum_{i=1}^{n}\left(f_{i} \frac{U_{i}^{T} \bar{D}}{\sigma_{i}}\right)^{2}$.

So,

$\left\|G s_{\alpha}-\bar{D}\right\|_{2}^{2}=\sum_{i=1}^{n}\left(\left(1-f_{i}\right) U_{i}^{T} \bar{D}\right)^{2}$,

where $f_{i}$ is the so called Tikhonov filter factors, defined as:

$f_{i}=\frac{\sigma_{i}^{2}}{\sigma_{i}^{2}+\alpha^{2}}$.

Such expressions (Eq. 9 and 10) can be used to illustrate what happens to the solution in the presence of noise. So, when adding uncorrelated noise $\eta$, this would result in extra contribution to the solution caused by noise components. Then, the true solutions $s_{o}$ can be expressed as (Fortin and Wolkowics, 2004):

$\left\|s_{o}\right\|_{2}^{2}=\sum_{i=1}^{n}\left(\frac{U_{i}^{T} D}{\sigma_{i}}+\frac{U_{i}^{T} \eta}{\sigma_{i}}\right)^{2}$,

assuming that both $\mathrm{G}$ and s are invertible.

So, for small singular values these contributions will be very large, whenever the noise vector is not orthogonal to the corresponding singular vectors, $U_{i}$. This simply clarifies why the naive least squares solution is not meaningful and a regularized solution should be sought instead. Even if there 
is no noise, the problem is still there if the matrix has very small singular values (close to zero). It is required that $\left|U_{i}^{T} D\right|$ should decay faster than $\sigma_{i}$. This is known as a Picard condition. It is guaranteed that if such condition is satisfied, the least squares solution has a reasonable norm and is thus physically meaningful (Hansen, 1999). Then, TRS can be used to form the L-curve (Fortin and Wolkowics, 2004) as:

$L(G, \bar{D}):=\left\{\left(\log (\varepsilon), \log \left\|G s_{\varepsilon}-\bar{D}\right\|\right): \varepsilon>0, \quad s_{\varepsilon}\right.$ is optimal for TRS $\}$.

As we are now familiar with the TRS and L-curve concepts, it is time to demonstrate in details the proposed PTRS method and briefly the well known TSVD with L-curve pruning.

\subsection{PTRS and TSVD regularization techniques}

PTRS method is an extension of the traditional TRS approach. Grodzevich (2004) has explained the PTRS method as an effective tool that can be used in conjunction with the L-curve maximum curvature criterion. The classical algorithm for solving TRS, with a given trust-region radius $\varepsilon$, is based on solving Eq. 4 for various choices of $\alpha$, and using a Cholesky factorization of $G^{T} G+\alpha^{2} I$ (Moré and Sorensen, 1983). More recently, a parameterized trust-region approach to find the regularized solution $x_{\varepsilon}$, has been used by Rojas and Sorensen (2002). Then, Grodzevich and Wolkowics (2009) have extended the traditional TRS approach that makes use of L-curve maximum curvature criterion, which is applied here to solve our problem. It is assumed that the easy case holds, and that the optimum point lies on the boundary of the feasible region, i.e. $\left\|s^{*}\right\|=\varepsilon$. Grodzevich and Wolkowics (2009) also showed that the TRS algorithm visits, each iteration, a point on the L-curve. Therefore, the trust-region radius $\varepsilon$ can be dynamically changed to steer the algorithm to the elbow of the L-curve. For further details on the method, the reader can refer to Grodzevich and Wolkowics (2009).

As the kernel function decays with the inverse squared depth, the inverted model will mostly be concentrated near the surface. This tendency can be overcome by introducing a depth weighting to counteract the natural geometric decay. Li and Oldenberg (1998) have shown that it was reasonable to approximate the decay with depth by a function of the form $w(z)=$ 
$1 /\left(z+z_{0}\right)^{\beta / 2}$, where $\beta$ is usually equal to 2 and $z_{0}$ depends upon a reference level. We used the diagonal matrix:

$W_{m_{i}}=1 /\left(z_{m_{i}}+\varepsilon\right)^{\beta}$,

where $\beta=0.9$ (Boulanger and Chouteau, 2001) and a small $\varepsilon$ to prevent singularity when $z$ is close to 0 .

To compare the inverted solutions (magnetic susceptibilities) to the true ones in synthetic examples; the Pearson correlation coefficient $(r)$ of two set of observations $\left\{\left(\mathrm{x}_{\mathrm{i}}, \mathrm{y}_{\mathrm{i}}\right): \mathrm{i}=1, \ldots, \mathrm{n}\right\}$ is used where

$r_{\text {corr }}=\frac{\sum_{i=1}^{n}\left(x_{i}-\bar{x}\right)\left(y_{i}-\bar{y}\right)}{\sqrt{\sum_{i=1}^{n}\left(x_{i}-\bar{x}\right)^{2} \sum_{i=1}^{n}\left(y_{i}-\bar{y}\right)^{2}}}$,

$\bar{x}$ and $\bar{y}$ are the mean values of $x$ and $y$, which represent synthetic and inverted susceptibilities in our case. When the two sets of data are identical, $r$ is unity, while they differ when $r<<1$.

The above method is used to invert the Earth's magnetic field. The inverted profile is assumed to be taken over an array of prismatic cells with different unknown magnetic susceptibilities. The complete code is written in Matlab 2009a and the function of the PTRS regularization tool, Grodzevich (2004), is implemented. The inputs are the number of rows and columns of the subsurface model, the width of each cell, vector of true susceptibilities for synthetic example, noise ratio is introduced to synthetic data as well as the magnetic parameters like the inclination, declination, total field etc. The code calculates the magnetic field of the synthetic model and adds also the required noise. The output is the inverted susceptibilities of the subsurface cells. The number of data points in real examples is usually less than the number of unknowns. Therefore, an interpolation function is used to increase the data in order to get an even or over-determined problem. Finally, the inverted model is drawn using a color scale to present the distribution of subsurface susceptibilities.

For the sake of comparison, the L-curve pruning with adaptive L-curve method is chosen to invert the same earth models as a robust tool described by Hansen et al. (2007). It has been applied successfully on gravity illposed inverse problem (Abdelazeem et al., 200\%). Its idea simply is to locate 
the corner of a discrete L-curve using truncated singular value decomposition (TSVD). A sequence of pruned L-curves is used to capture the global features of the curve. The main idea was to remove the right amount of points from the discrete L-curve, so the corner can easily be found using the remaining set of points. For more details refer to the above two papers.

\section{Synthetic examples}

The new PTRS method (Grodzevich and Wolkowics, 2009) is tested using two synthetic examples contaminated with different noise levels. The TSVD method with L-curve pruning (Hansen et al., 2007) is also applied to the same examples for comparison.

\subsection{Example 1}

The first synthetic example (Model 1) consists of 160 square cells arranged in 8 rows and 20 columns. The ambient field is assumed to be $40000 \mathrm{nT}$ with a direction of inclination $60^{\circ}$ (same as inclination of cell magnetization) and declination $3^{\circ}$. The Azimuth of the measured data is taken as zero. The data points are 160, i.e. our problem is an even-determined one. The condition number of matrix $\mathrm{G}$ is found to be $1.6932 \cdot 10^{12}$. As the noise free data is not an actual case, we start with low perturbed data (Signal to Noise Ratio, SNR $=90 \mathrm{~dB}$ ). The Picard condition is tested for noise free data (Fig. 1a) and also for highly perturbed one (Fig. 1b), $15 \mathrm{~dB}$, to illustrate the effect of noise on Picard plot. The Picard condition is satisfied till the first 80 prisms (in noise free case) and till the first 60 prisms (when $15 \mathrm{~dB}$ noise is added), where the singular values $\sigma$ decay faster than the Fourier coefficients. The singular values decay from the order of $10^{5}$ to the order of $10^{-7}$ causing the condition number to be of the order of $10^{12}$.

Different noise levels have been added to the data and the problem is solved using both the proposed PTRS method and TSVD with pruning L-curve for comparison. The inverted earth models together with the observed and inverted magnetic fields are shown in Figs. 2 and 3. The Earth's model is dramatically distorted using TSVD with pruning L-curve, when $\mathrm{SNR}=60 \mathrm{~dB}$, is added to data. On the other hand, the PTRS method has 

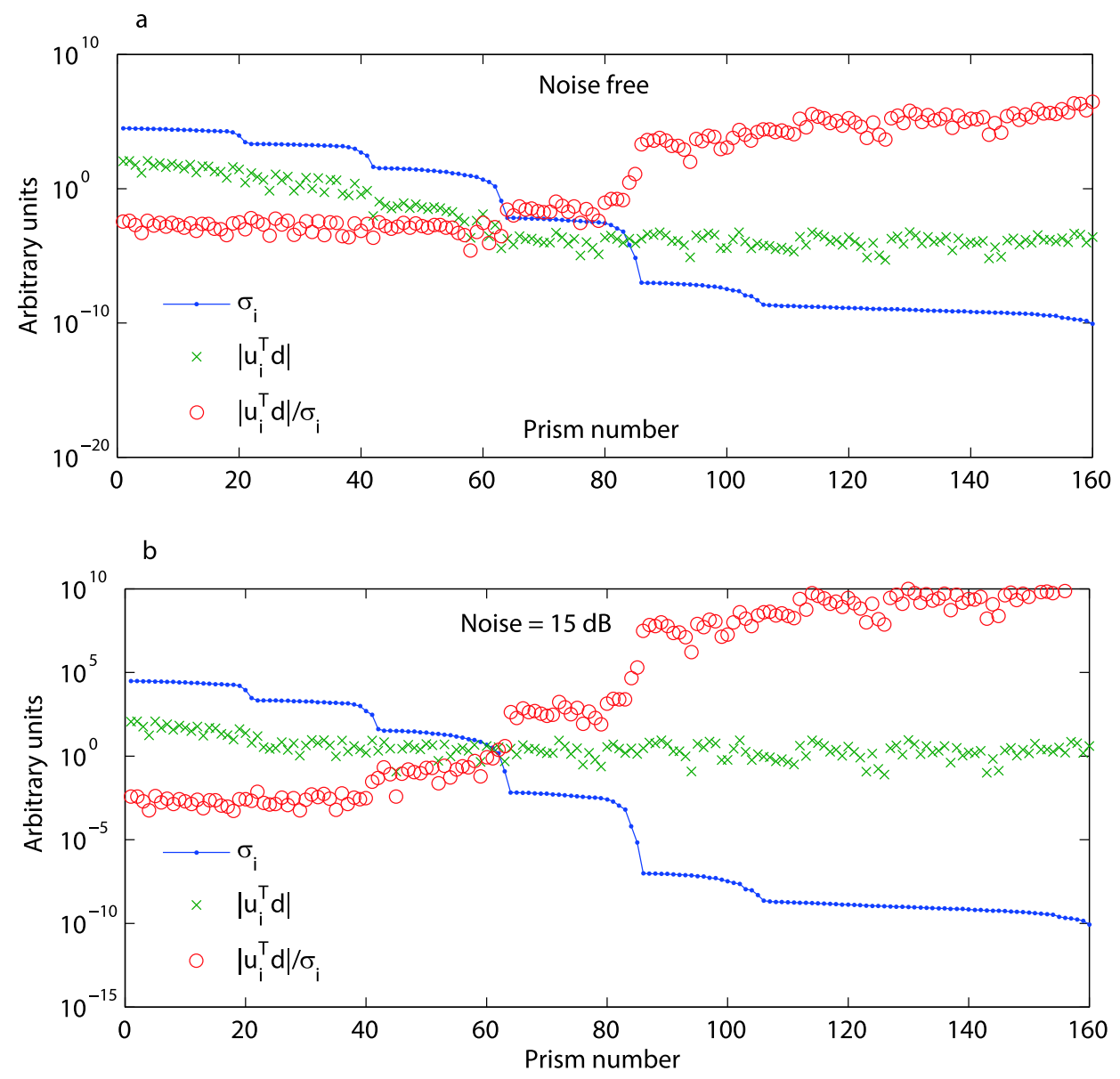

Fig. 1. The Picard plot for synthetic example 1, a) for noise free and b) for $15 \mathrm{~dB}$ noise level implemented.

been applied successfully to the same model with noise ratio varying from $80 \mathrm{~dB}$ to $15 \mathrm{~dB}$, i.e. higher noise has been added. Subsequently, the Pearson correlation coefficient $r_{\text {corr }}$ calculated between the inverted and actual data are nearly complete coincidence (0.99 to 0.98 ) for both methods. Also, the correlation coefficient is calculated between the inverted susceptibilities (solutions) and the actual values, which is presented in Table 1 for different noise ratios. It slightly decreases with increasing noise using the PTRS 

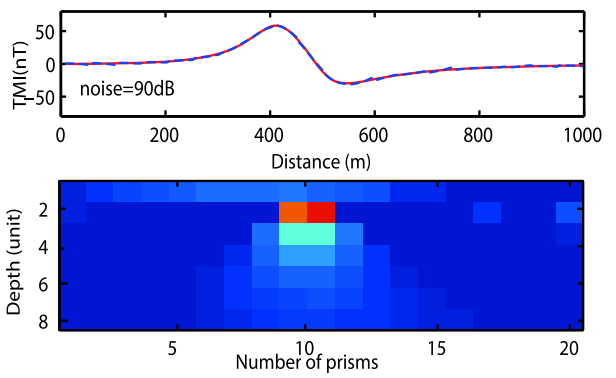

C
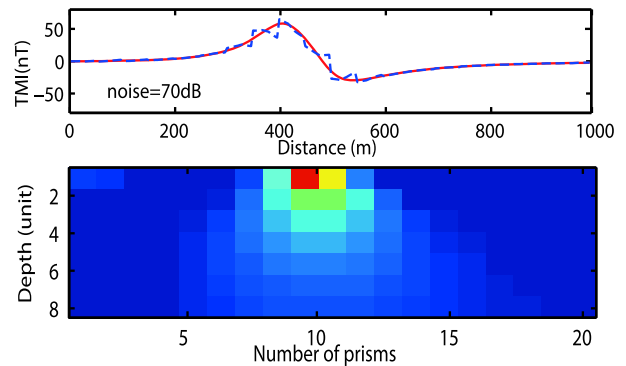

e
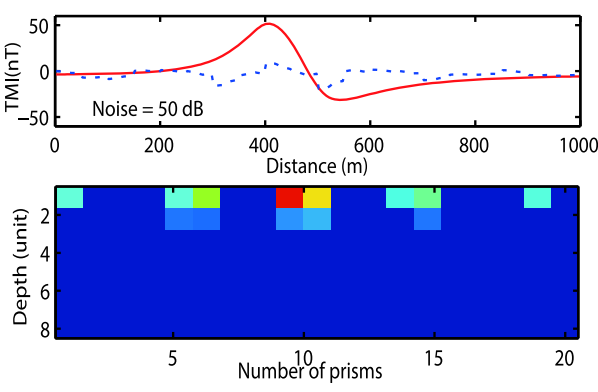

1 unit $=50 \mathrm{~m}$ b
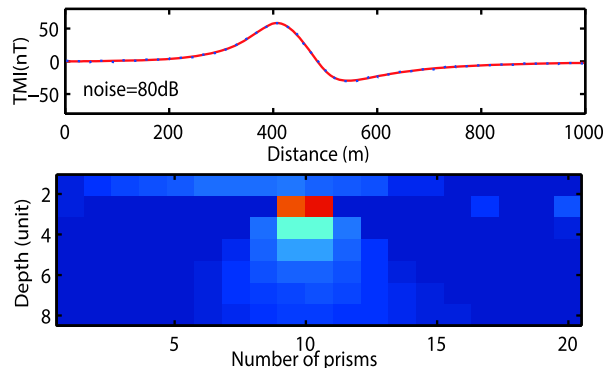

d
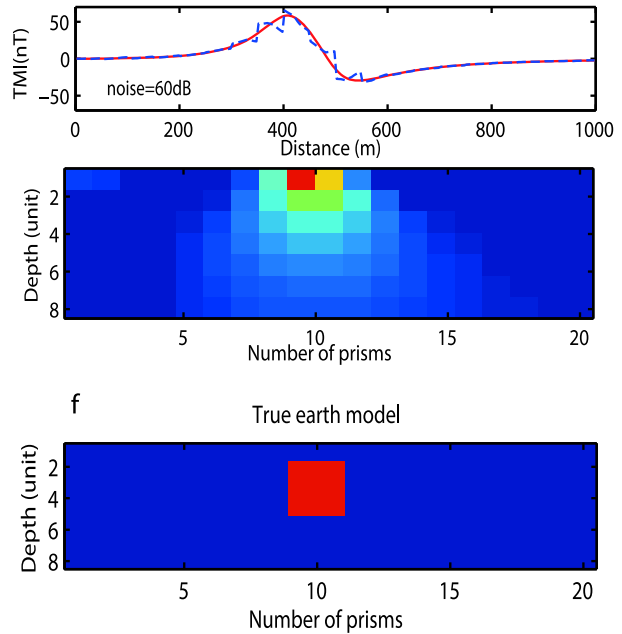

Magnetic susceptibility

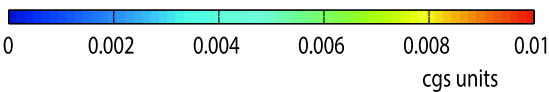

Observed

Inverted

Fig. 2. Inverted earth models, using TSVD with L-curve pruning, together with true and inverted magnetic fields when adding different noise levels to data, a) $90 \mathrm{~dB}, \mathrm{~b}) 80 \mathrm{~dB}, \mathrm{c}$ ) $70 \mathrm{~dB}$, d) $60 \mathrm{~dB}$ to data, e) $50 \mathrm{~dB}$ and f) True model.

method. When the same model was inverted using TSVD with adaptive pruning L-curve (Hansen et al., 2007 and Abdelazeem et al., 200\%), the inverted model was acceptable, nevertheless the two bottom rows of prisms 
a
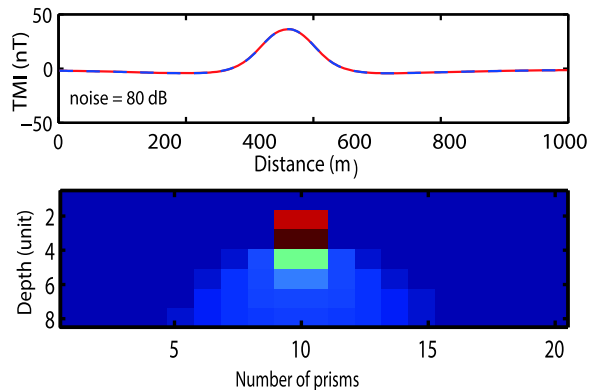

C
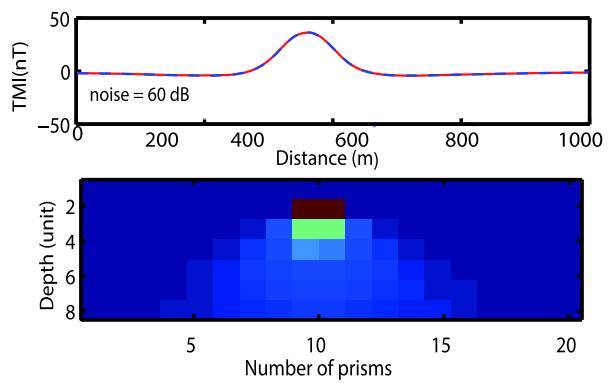

e
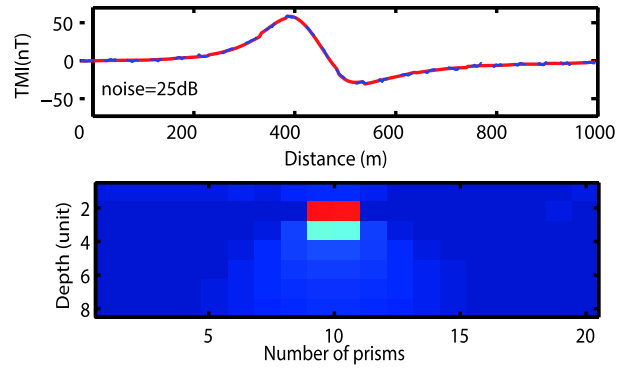

1 unit $=50 \mathrm{~m}$ b
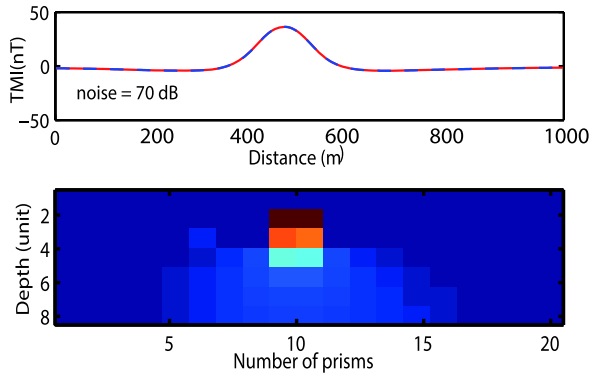

d
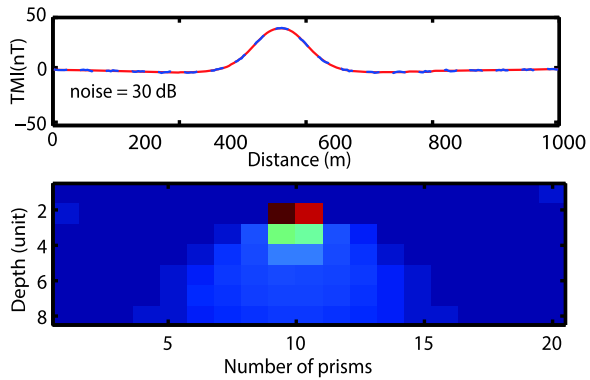

$f$
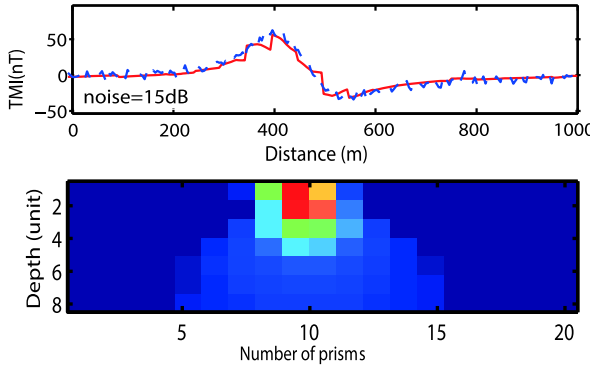

Observed -.-.--Inverted

Fig. 3. Inverted earth models, using PTRS, together with true and inverted magnetic fields when adding different noise levels to data, a) $80 \mathrm{~dB}$, b) $70 \mathrm{~dB}$, c) $60 \mathrm{~dB}$ and d) 30 $\mathrm{dB}$ e) $25 \mathrm{~dB}, \mathrm{f}) 15 \mathrm{~dB}$ to data and g) the true earth model.

attain very high susceptibilities, which dominated the rest of the plausible model. The correlation factors between inverted susceptibilities using PTRS and TSVD with pruning L-curve and true ones are presented also in Table 1. 
Table 1. The Pearson correlation coefficient between true and inverted vectors of magnetic susceptibilities resulted from PTRS and TSVD with pruning L-curve for model 1, using different SNR

\begin{tabular}{ccc}
\hline \multirow{2}{*}{ SNR (dB) } & $\boldsymbol{r}_{\text {corr }}$ between inverted and true susceptibilities \\
\cline { 2 - 3 } & PTRS & TSVD \\
\hline 90 & 0.90 & 0.75 \\
80 & 0.90 & 0.75 \\
70 & 0.89 & 0.45 \\
60 & 0.80 & 0.09 \\
50 & 0.80 & 0.09 \\
30 & 0.79 & - \\
25 & 0.78 & - \\
15 & 0.58 & - \\
\hline
\end{tabular}

Notice the dramatic decrease in the correlation factor with increasing noise, i.e. when decreasing SNR.

\subsection{Example 2}

The second synthetic example (Model 2) represents two bodies, an outcropping inclined dike and a buried massive body. The number of square cells $(\mathrm{M})$ is 160 and the data points $(\mathrm{N})$ are 200 . The ambient field $F$ is assumed to be $40000 \mathrm{nT}$, the inclination of the field is $60^{\circ}$, and the declination is $2^{\circ}$. The inclination of all prisms is assumed to be identical and equal to the field inclination. Magnetization is assumed to be induced, but the program is designed to hold the remnant magnetization if it exists. The condition number of the kernel matrix is found to be $5.487910^{14}$. The Picard plot is shown in Fig. 4 for both noise free and contaminated data, 15 dB. Figs. 5a, $\mathrm{b}, \mathrm{c}$ and $\mathrm{d}$ show the TSVD solutions for data contaminated with SNR of $90 \mathrm{~dB}$ to $60 \mathrm{~dB}$. True model is shown in Fig. 5e. On the other hand, the PTRS method is applied using SNR from $80 \mathrm{~dB}$ to $15 \mathrm{~dB}$ on the same data. The results (Fig. 6) are accepted till $20 \mathrm{~dB}$ noise level, as the depth to top is accepted. When $15 \mathrm{~dB}$ has been added to the data, the depth to top becomes nearer to surface. Table 2 shows that the correlation coefficient 
between true and inverted models by PTRS are closer to unity, while those inverted by TSVD with pruning L-curve ranges from 0.75 for $90 \mathrm{~dB}$ SNR to 0.09 for $60 \mathrm{~dB}$ SNR. These results prove the stability of PTRS with higher levels of noise.

Table 2. Summarizes the results of model 2 through different noise levels and the corresponding change in Pearson correlation factor between true and inverted vectors (magnetic susceptibilities) using PTRS and TSVD with L-curve pruning

\begin{tabular}{ccc}
\hline SNR (dB) & \multicolumn{2}{c}{$\begin{array}{c}\text { Correlation between inverted and } \\
\text { true magnetic susceptibility vectors }\end{array}$} \\
\cline { 2 - 3 } & PTRS & TSVD (pruning) \\
\hline 90 & 0.91 & 0.75 \\
80 & 0.91 & 0.75 \\
70 & 0.90 & 0.45 \\
60 & 0.88 & 0.09 \\
50 & 0.81 & 0.09 \\
40 & 0.81 & - \\
25 & 0.80 & - \\
20 & 0.79 & - \\
15 & 0.63 & - \\
\hline
\end{tabular}

\section{Field examples}

PTRS method has been further applied to a real total magnetic field data from Saudi Arabia over Al Ji'lani basic intrusion (example 1), and a vertical gradient magnetic field of an archeological site in Italy (example 2).

\subsection{Field example 1}

The Precambrian Al Ji'lani basic intrusion forms an oval outcrop $(9 \times 6 \mathrm{~km})$ over $50 \mathrm{~km}^{2}$ of relatively flat well-exposed country, $10 \mathrm{~km}$ southwest of Ad Dawadimi district, Kingdom of Saudi Arabia. Within the oval-shaped outcrop area of the intrusion, there is a total exposed thickness of $2,600 \mathrm{~m}$ of rock which dips inward, and consists of amphibolitized and fresh gabbros, 

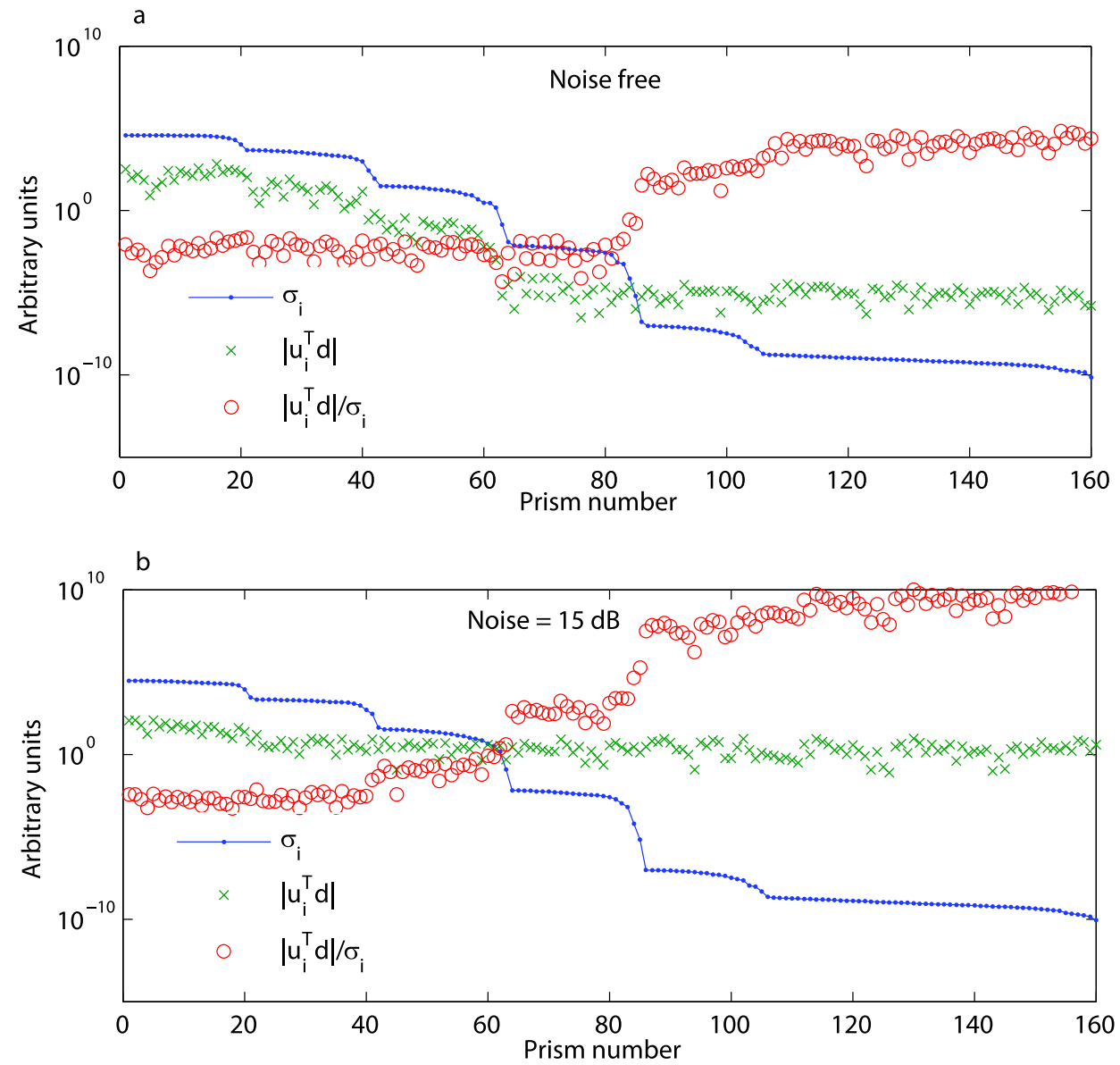

Fig. 4. Picard plot for example 2, (a) for noise free data, and b) for $15 \mathrm{~dB}$ noise level implemented.

troctolites, norites, and anorthosites (Al Shanti, 1974). The Al Ji'lani layered intrusion has its major axis trends $\mathrm{N} 42^{\circ} \mathrm{W}$. The intrusion is made up of rather uniform concentric layers dipping radially inward. Dips are generally steep, the marginal layers dipping $50-70^{\circ}$ and the central layers dipping about $20^{\circ}$. Local distortions in dips and layering are due to later dike and granitic intrusions as displayed in the central area.

The layered intrusion has a marked, well defined, aeromagnetic signa- 

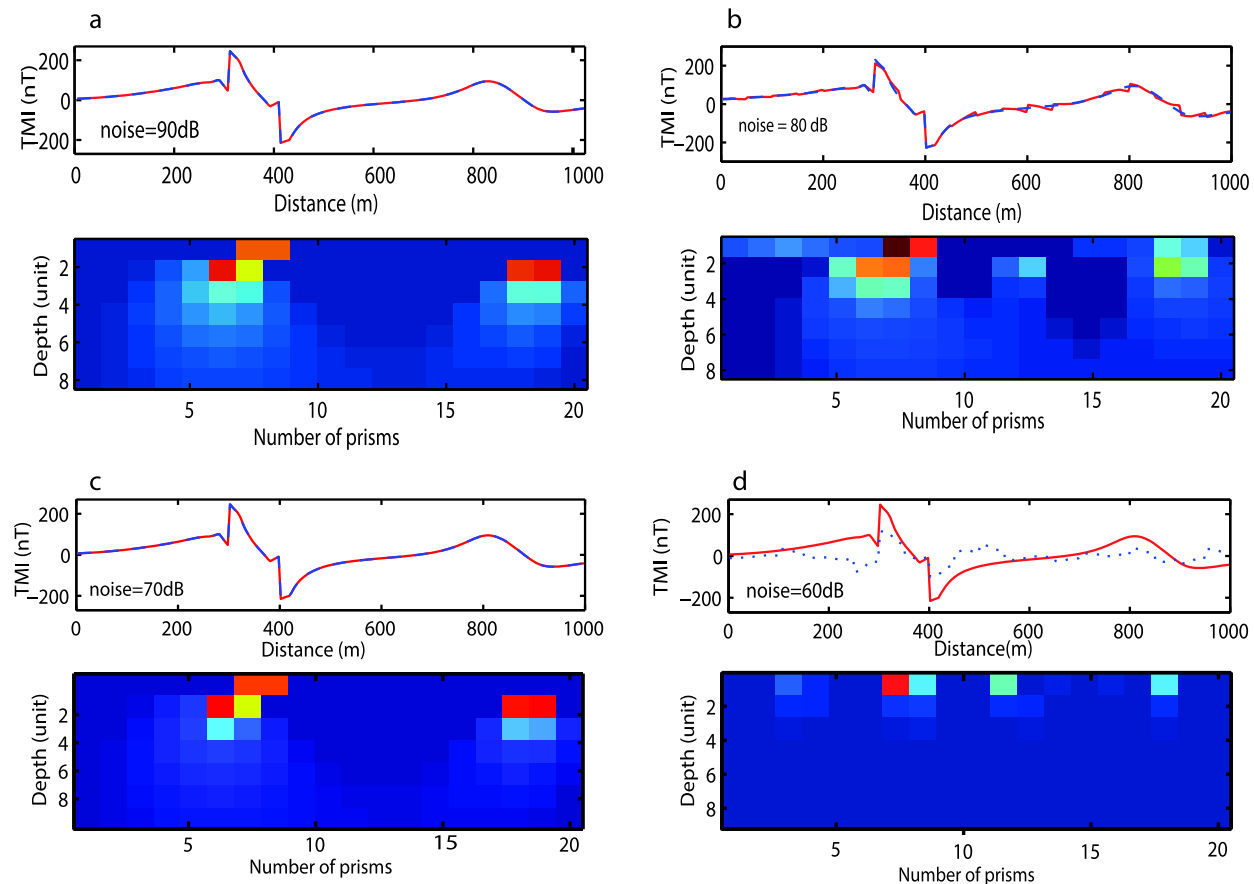

1 unit $=50 \mathrm{~m}$

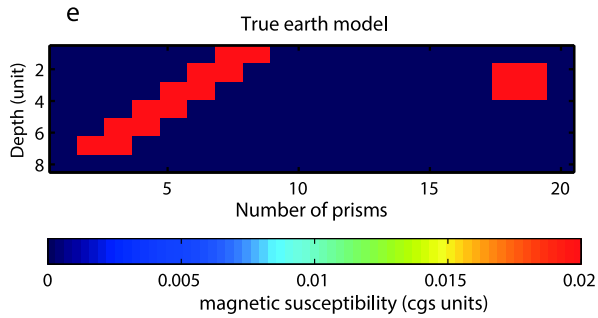

Observed -------Inverted

Fig. 5. Inverted earth models, using TSVD with adaptive pruning L-curve, together with true and inverted magnetic fields when adding different noise levels to data, a) $90 \mathrm{~dB}$, b) $80 \mathrm{~dB}$, c) $70 \mathrm{~dB}, \mathrm{~d}) 60 \mathrm{~dB}$ to data and e) the true earth model.

ture within the surrounding area of low magnetic relief, as shown in Fig. 7. Lambolez (1968) qualitatively interpreted the magnetic pattern to indicate a deep-rooted basic mass with a rather sharp and steeply dipping contact and noted the presence of marked subsidiary anomalies within the structure. The suggested structure of a steep-sided mass is important relative to the alternative possibility that the intrusion has a shallow lopolithic floor ad- 

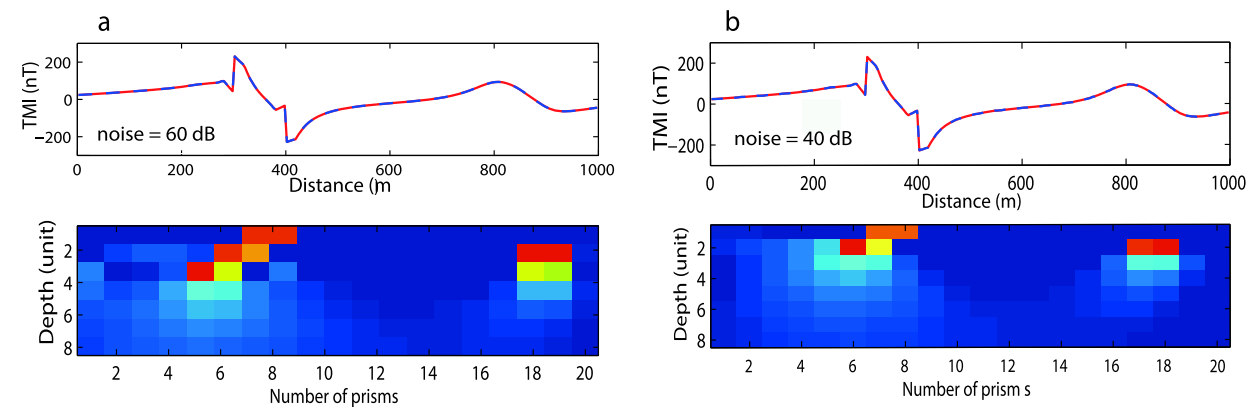

C

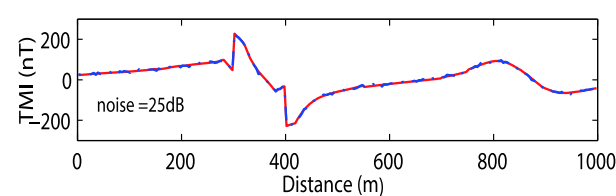

d
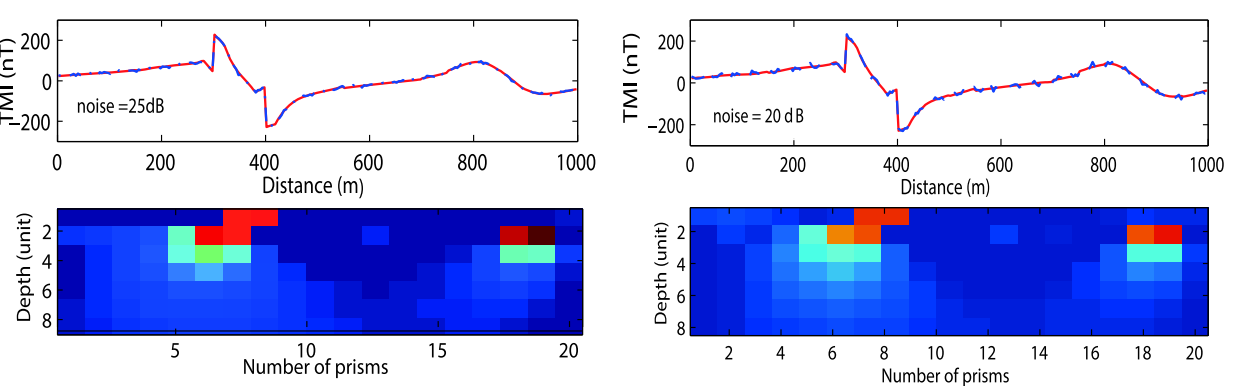

e
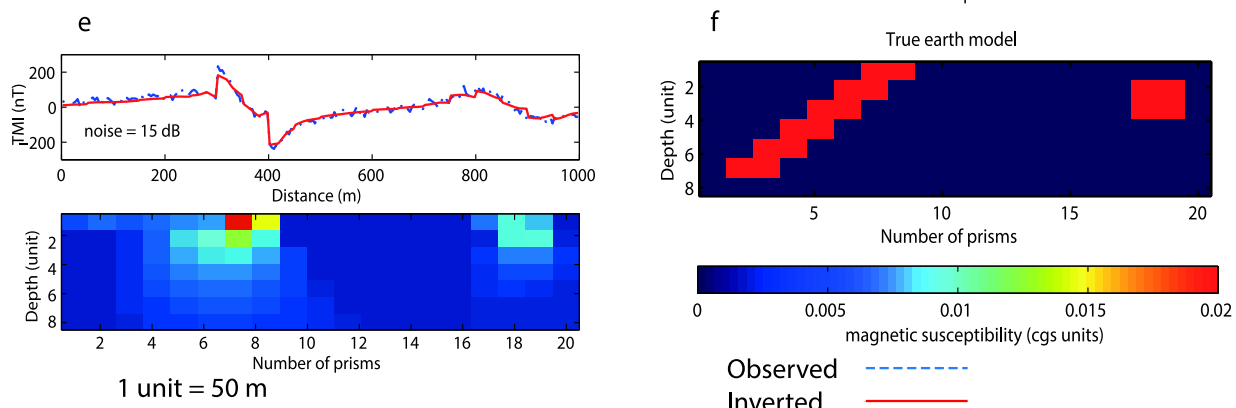

Observed ------Inverted

Fig. 6. Inverted earth models, using PTRS method, together with true and inverted magnetic fields when adding different noise levels, a) $60 \mathrm{~dB}$, b) $40 \mathrm{~dB}$, c) $25 \mathrm{~dB}$ and d) $20 \mathrm{~dB}$ e) $15 \mathrm{~dB}$ to data and f) The true model with color scale used for susceptibility.

jacent to the outermost ring of basic rock. Present geologic investigations suggest roof pendent intrusion instead of deep rooted mass. The PTRS technique has been applied to reveal the present doubt.

The area of study and the studied profiles A-A' and B-B' are shown in Fig. 7. Declination of the area is $2^{\circ} 35^{\prime}$ and the total field is $40000 \mathrm{nT}$. 


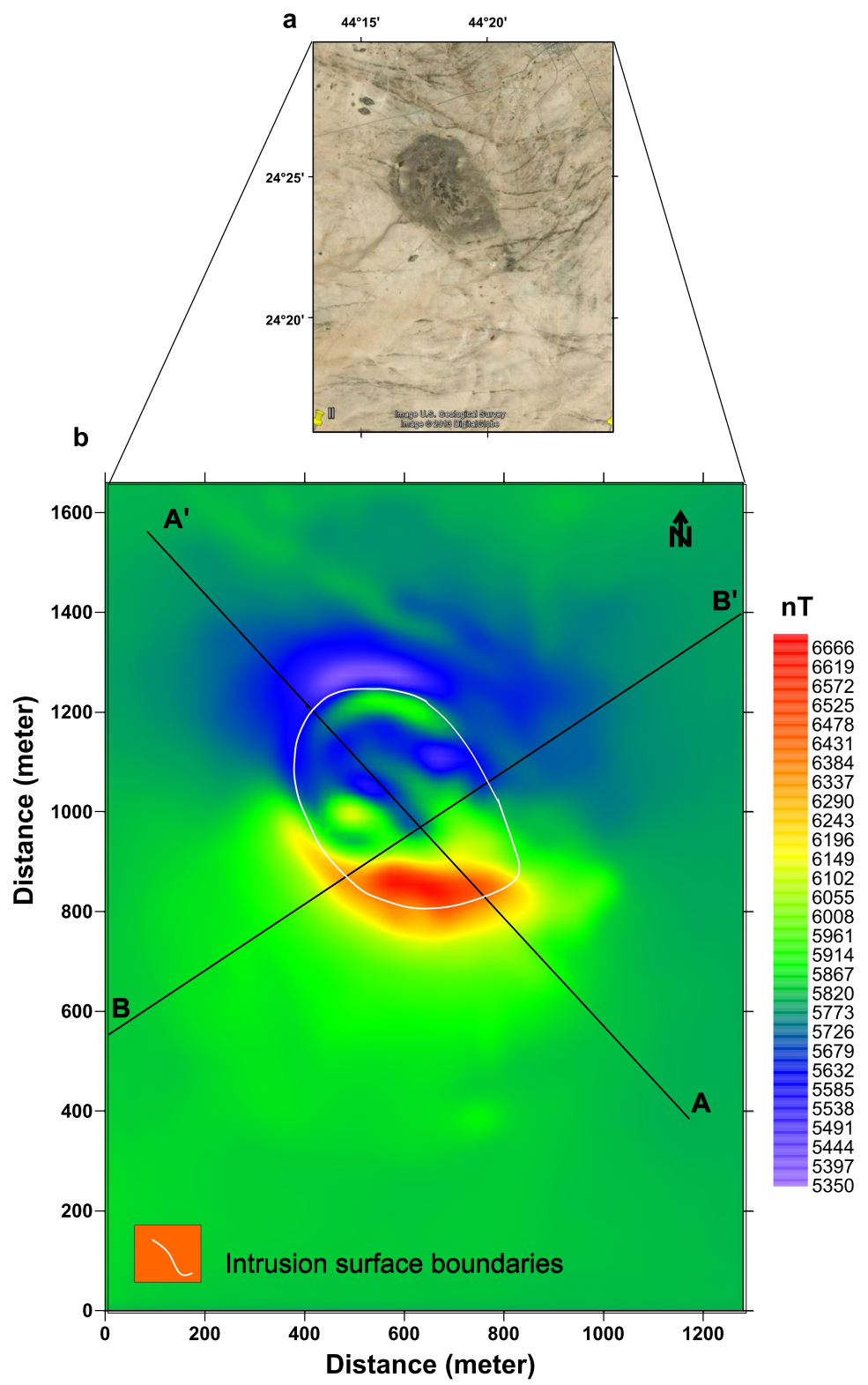

Fig. 7. Al Ji'lani intrusion, Saudi Arabia. a) Location map with a satellite image retrieved from Google Earth. b) The RTP magnetic over the area under study with the two profiles subjected to inversion. 
a
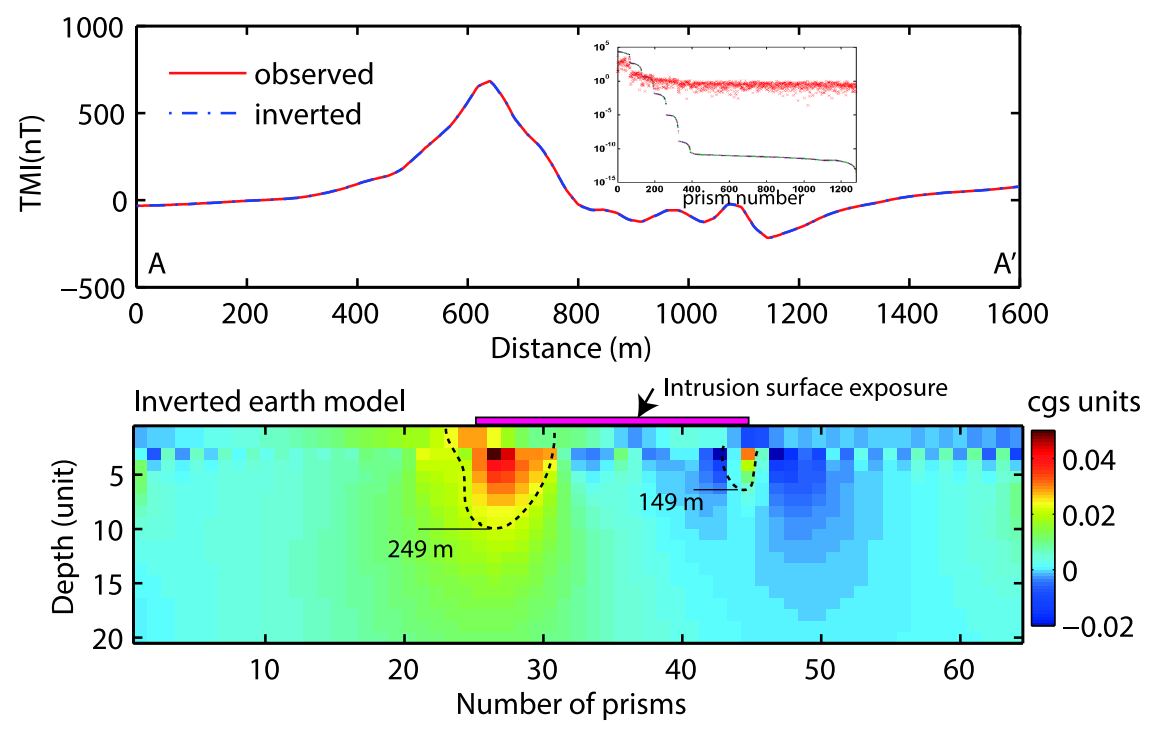

b
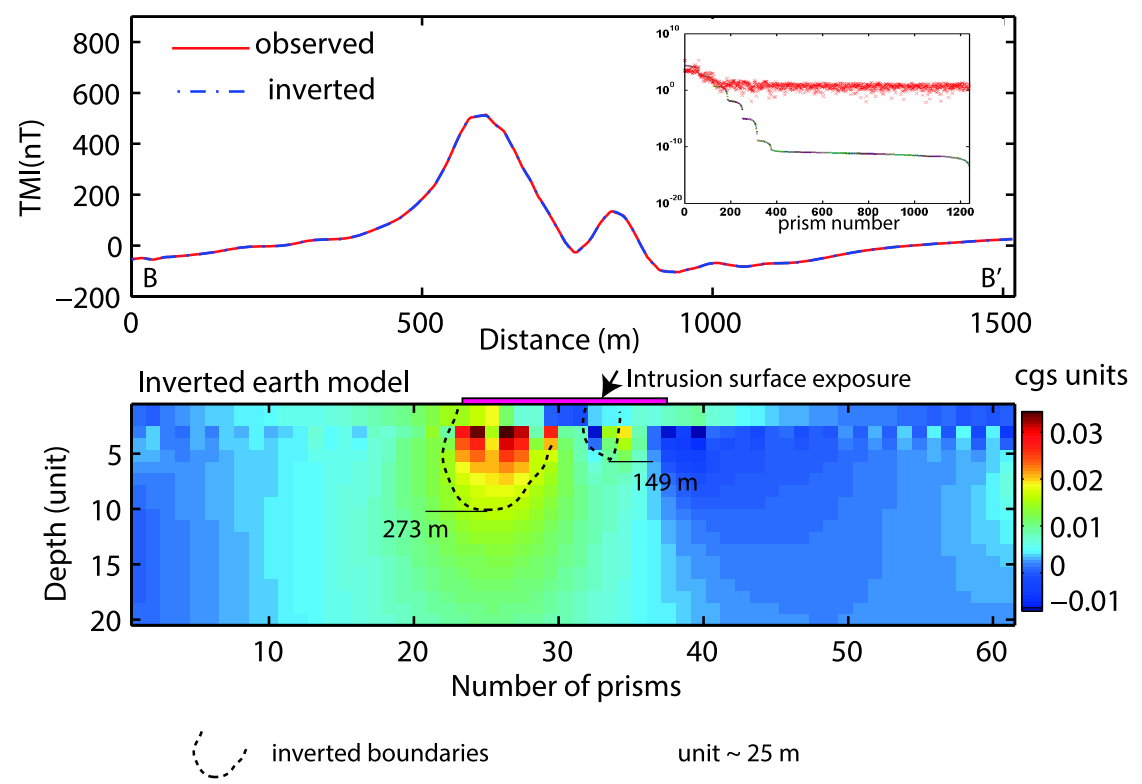

Fig. 8. Inversion results for field example 1 over a) profile A-A' and b) profile B-B'. Picard plots are shown as sub-plots. 
The regional trend is subtracted from profiles A-A' and B-B' before inversion tool is used. Fig. 8a shows the profile A-A' and the inverted model together with the Picard plot as a sub-plot. The profile A-A' is of total length $1598 \mathrm{~m}$. The model Earth is subdivided into 1280 square cells (M), arranged in 20 rows and 64 columns. The data points $(\mathrm{N})$ are interpolated to 1280 data points. The Picard plot proves the ill-posedness of the problem represented by the fast decaying of the $\sigma_{i}$ (condition number $\sim 10^{20}$ ) with respect to slower decay of $\left|U_{i}^{T} d\right|$. The Picard condition is only satisfied in the first 200 cells. Consequently, PTRS method is applied to solve this highly ill-posed problem. The inverted earth model suggests two bodies. One is to the left with maximum depth of $249 \mathrm{~m}$ and the other to the right with maximum depth of $149 \mathrm{~m}$. The profile B-B' is of total length $1515 \mathrm{~m}$. The earth model is subdivided into 1220 square cells arrange as 20 rows and 61 columns. So, the unit is nearly the same as that in first model $(\sim 25 \mathrm{~m})$. Inverted model over profile B-B' is shown in Fig. $8 \mathrm{~b}$ with its Picard plot also showing severe ill-posedness. The condition number $\sim 10^{20}$, which is more than that appeared in the synthetic examples. The inverted model, using PTRS method, suggests also two near bodies, the one to the left one extends to $273 \mathrm{~m}$ and the right one has a maximum depth of $149 \mathrm{~m}$. This result confirms the roof bendent intrusion hypothesis over the deep rooted base mass.

\subsection{Field example 2}

The PTRS has also been applied to magnetic data in a well-studied area of the Sabine Necropolis at Motelibretti, Rome, Italy (Stocco et al., 2009). This archeological site is characterized by chamber tombs, and we have to find its position and size. A N-S profile is extracted from the vertical gradient field of the vertical component map of the area and subjected to inversion using PTRS technique. Although no constraints are added except the method's powerful dynamic trust region estimation, the results show a remarkable compatibility to previous results (Stocco et al., 2009). Fig. 9a demonstrates Stocco's inversion results, whereas the inverted earth model using PTRS is shown in Fig. 9b. The ill-posedness of the problem is obvious from the Picard plot. The inversion results show a depth and shape of the two buried rooms very close to Stocco's model. 

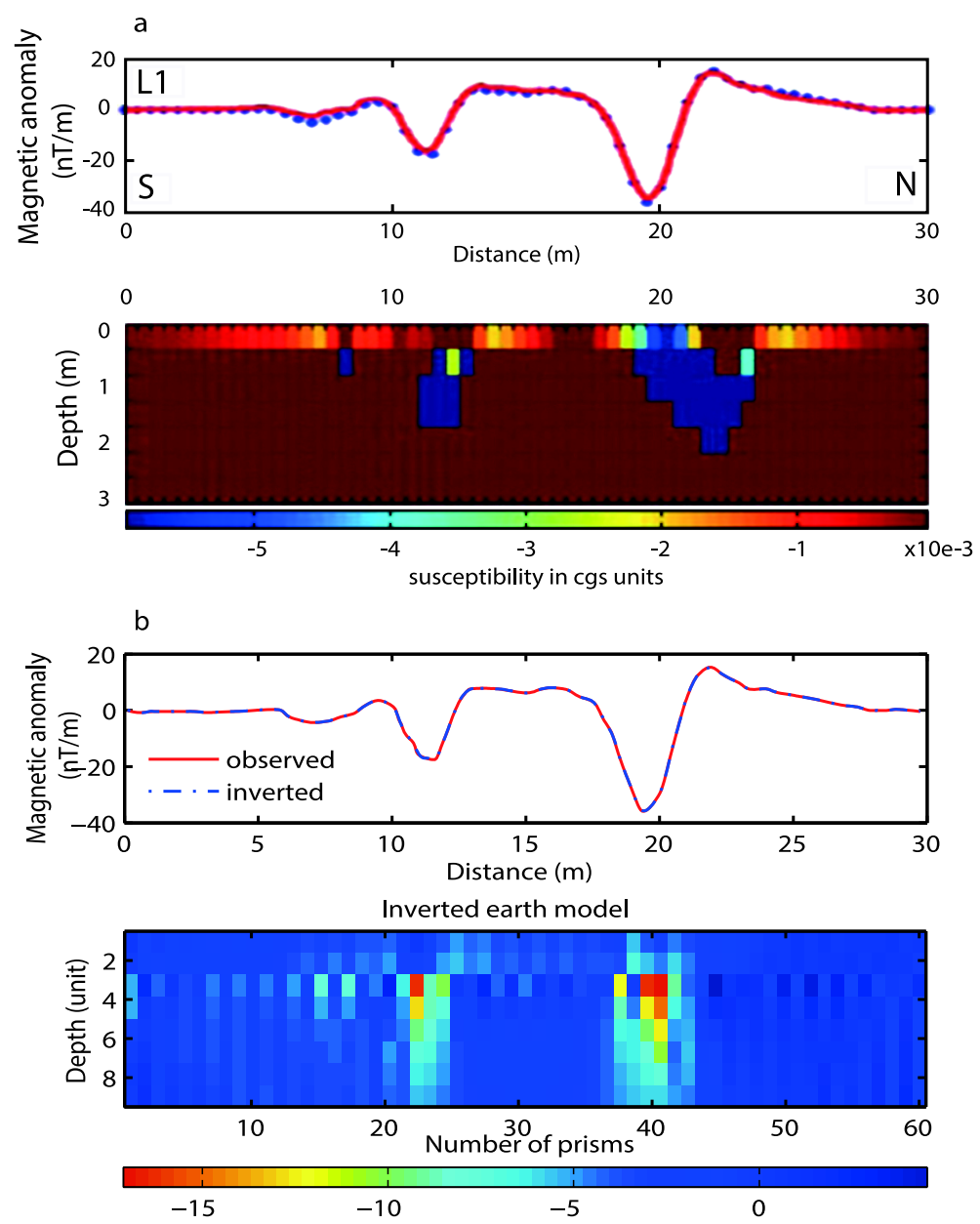

Fig. 9. Inversion results for field example 2 (Sabine Necropolis at Motelibretti, Rome, Italy). a) Inverted model of magnetic field profile over the Sabine Necropolis, at Montelibretti, Rome, Italy (Stocco, 2009). b) Inverted earth model using PTRS for the same profile.

\section{Conclusion}

Some magnetic inverse problems cannot be solved with standard inversion tools. This is due to instability, sensitivity to noise content in data or ill- 
conditioned kernel matrix. The ill-posedness of the problem appears when the condition number is in the order of $\sim 10^{5}$ or higher. The higher the condition number, the stronger the ill-posedness of the problem. This illposedness will appear clearly in the Picard plot represented by the fast decay of the $\sigma_{i}$ with respect to slower decay of $\left|U_{i}^{T} d\right|$. In such cases we should use regularizing techniques. In this paper a computationally efficient Parameterized Trust Region Sub-problem technique (PTRS) has been applied to the magnetic inverse problem. A comparison between TSVD with adaptive pruning L-curve technique and the newly proposed PTRS approach, using two synthetic examples with different noise levels, suggests higher stability of the PTRS against higher noise levels. As shown in the examples, the dynamically changed trust-region radius $\varepsilon$ is a key element to find the satisfying minimum to the objective function by steering the algorithm to the elbow of the L-curve.

Successful use of the proper weighting to the kernel function minimizes the tendency of the calculated model to cluster near the surface. PTRS approach appears to work well in practice as illustrated by application to different field examples of various degrees of ill-posedness.

In summary this inversion approach seems promising for real magnetic inverse problem solution where much noise content in the data is expected. The potential of this approach across a range of applications merits further testing on more complicated geologic problems.

Acknowledgments. I am grateful for Prof. Dr. Hanfy Debees from National Research Institute of Astronomy and Geophysics (NRIAG) for reviewing the manuscript. My great appreciation to Prof. Nasser Sweilam (Department of Mathematics) and Prof. Mohamed Gobashy (Department of Geophysics) from Cairo University for their constructive and helpful suggestions. Thanks also to Prof. El-Dogdog (King Abdulaziz University, Faculty of Earth Sciences) for the valuable geological discussion of the first field example. Sincere thanks go also to the reviewers and Editor, who helped me to improve the manuscript.

\section{References}

Abdelazeem M., Sweilam N., Bayoumi A. I., Refai E. M., 1998: Application of Linear Quadratic Programming to the Inverse Gravity Problem, Africa/Middle East. Second International Geophysical Conference \& Exposition, Cairo, Egypt, Feb. 17-19. 
Abdelazeem M., Sweilam N. H., Gobashy M., Nagy A. M. A., 2007: Two dimensional gravity inverse problem using adaptive pruning L-curve technique. Bull. Fac. Sci., Cairo Univ., 75, (A), 93-115.

Al Shanti A. M., 1974: Al Ji'lani layered basic intrusion, Ad Dawadmi District, Kingdom of Saudi Arabia. Mineral resources Bulletin, 12, Directorate General of Mineral Resources, Kingdom of Saudi Arabia.

Ardalan A. A., Zamzam D., Karimi R., 2011: An alternative method for density variation modeling of the crust based on 3-D inversion. Journal of Applied Geophysics, 75, $355-362$.

Baker C. T. H., 1977: The numerical treatment of integral equations. Clarendon Press, Oxford.

Blakley R. J., 1996: Potential theory in gravity and magnetic applications. Cambridge University press.

Blaschek R., Hördt A., Kemna A., 2008: A new sensitivity-controlled focusing regularization scheme for the inversion of induced polarization data based on the minimum gradient support. Geophysics, 73, F45-F54.

Boulanger O., Chouteau M., 2001: Constraints in 3D gravity inversion. Geophys. Prospect., 49, 265-280.

Dennis J. E. Jr., Schnabel B. R., 1983: Numerical methods for unconstrained optimization and nonlinear equations. Prentice-Hall, Englewood Cliffs, New Jersey.

Fedi M., Hansen P. C., Paoletti V., 2005: Analysis of depth resolution in potential-field inversion. Geophysics, 70, A1-A11.

Fortin C., Wolkowics H., 2004: The trust region sub-problem and semidefinite programming. Optimization methods and software, 19, 41-67. (Special issue dedicated to Jochem Zowes $60^{\text {th }}$ birthday, Guest editors: Florian Jarre and Michal Kocvara).

Gander W., 1978: On the linear least squares problem with a quadratic constraints. Technical report STAN-CS-78-697, Department of Computer Sciences, Stanford University, Stanford, CA.

Grodzevich O., 2004: Regularization using Parameterized Trust Region Sub-problem. Master Thesis in mathematics, University of Waterloo, Ontario, Canada.

Grodzevich O., Wolkowicz, H., 2009: Regularization using parameterized trust region sub-problem. Math. Program., Ser. B, 116, $\overline{193}-\overline{2} 20$.

Guillen A., Menichetti V., 1984: Gravity and magnetic inversion with minimization of a specific functional. Geophysics, 49, 1354-1360.

Hansen P. C., 1990: The Discrete Picard Condition for discrete ill-posed problems. BIT, 30, 658-672.

Hansen P. C., 1998: Rank-deficient and discrete ill-posed problems, numerical aspects of linear inversion. Technical university of Denmark, Lyngby, SIAM, Philadelphia.

Hansen P. C., 1999: The L-curve and its use in the numerical treatment of inverse problems. Technical report, Technical University of Denmark.

Hansen P. C., Jensen T. K., Rodrigues G., 2007: An adaptive pruning algorithm for the discrete L-curve criterion. Journal of computational and applied mathematics, 198, 483-492. 
Lambolez B., 1968: Aeromagnetic and scintillometric survey 1966-67; Report on starting operations. Saudi Arabian Gen. Mineral Resources open-file rept.

Last B. J., Kubik K., 1983: Compact gravity inversion. Geophysics, 48, 713-721.

Li Y., Oldenburg D. W., 1998: 3-D inversion of gravity data. Geophysics, 63, 109-119.

Moré J. J., Sorensen D. C., 1983: Computing a trust region step. SIAM J. Sci. Stat. Comput., 4, 3, 553-572.

Morse P. M., Fashbach H., 1953: Methods of theoretical physics. McGraw-Hill, New York.

Pašteka R., Karcol R., Kušnirák D., Mojzeš A., 2012: REGCONT, A Matlab based program for stable downward continuation of geophysical potential fields using Tikhonov regularization. Computer and Geosciences, 49, 278-289.

Portniaguine O., Zhdanov M. S., 2002: 3-D magnetic inversion with data compression and image focusing. Geophysics, 67, 1532-1541.

Rojas M., Sorensen D., 2002: A trust region approach to the regularization of large-scale trust region sub-problem. M. SIAM J. Sci. Comput., 23, 6, 1842-1860 (electronic).

Silva J. B. C., Oliveira F. S., Barbosa V. C. F., Velho H. F. C., 2007: Apparent density mapping using entropic regularization. Geophysics, 72, 4, 151-160.

Stocco S., Godio A., Sambuelli L., 2009: Modelling and compact inversion of magnetic data: A Matlab code. Computers and Geosciences, 35, 2111-2118.

Tezkan B., Hoerdt A., Gobashy M., 2000: Two dimensional radiomagnetotelluric investigation of industrial and domestic waste sites in Germany. Journal of Applied Geophysics, 44, 2-3.

Tikhonov A. N., Arsenin V. Y., 1977: Solutions of ill-posed problems. V. H. Winston \& Sons.

Zhdanov M. S., 1993: Regularization in inversion theory: Tutorial, Colorado School of mines. 